\title{
ESTUDIO RADIOLÓGICO SOBRE LA FRECUENCIA DE PRESENTACIÓN DE DISPLASIA DE CADERA EN CANINOS DE LA RAZA LABRADOR RETRIEVER
}

\author{
Juan Tong G. ${ }^{1}$, Víctor Fernández A. ${ }^{2}$, Jorge Mendoza A. ${ }^{3}$ y Diego Díaz C. ${ }^{2}$
}

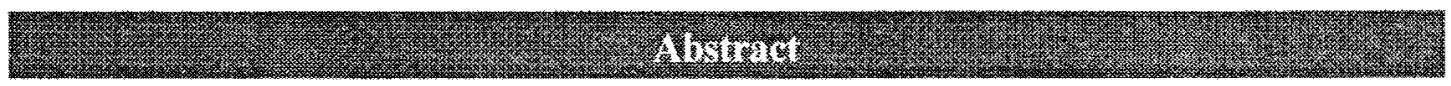

The incidence of hip displasia in 60 Labrador Retrievers was evaluated at the Small Animal Clinic, Faculty of Veterinary Medicine, San Marcos University, Lima, following radiological procedures dictated by the Federación Cinológica Internacional $(\mathrm{FCl})$. Two groups, comprised of 30 male and 30 female dogs, were formed at random and data on sex, age, clinical symptoms and coat color were recorded. Measurement of the Norberg angle in x-rays of both coxofemoral joints was used to determine the presence or absence of malformations. Hip displasia was diagnosed in $61.7 \%(37 / 60)$ of the cases, an elevated incidence compared to data from other countries. Both sexes were equally affected (no statistically significant difference) and most of the displasias were bilateral. Because only $48.64 \% \pm 16.10$ of the affected animals exhibited clinical symptoms, it is clear that radiological study is essential for precise and definitive diagnosis of canine hip dysplasia. Key words: Hip dysplasia, canines, radiology, Norberg angle, coxofemoral joint.

\section{Revinen}

El presente trabajo es un estudio radiológico que evalúa la presencia de displasia de cadera (D.C. ó D.C.F.) en las articulaciones coxofemorales de caninos de la raza Labrador Retriever. Este estudio ha sido desarrollado según los lineamientos científicos requeridos por la Federación Cinológica Internacional (FCI) para asuntos concernientes a dicha enfermedad, que rigen en la mayoría de países, incluyendo Perú. Los ejemplares fueron evaluados en la Clínica de Animales Menores de la Facultad de Medicina Veterinaria de la Universidad Nacional Mayor de San Marcos. Se estudiaron 60 animales escogidos al azar y distribuidos en dos grupos según el sexo ( 30 en cada uno). Se registraron datos como sexo, edad, presencia de sintomatología clínica, color del pelaje, y ángulo de Norberg en cada articulación coxofemoral para determinar radiológicamente la presencia o ausencia de la enfermedad. A partir del estudio radiológico se determinó que la frecuencia de presentación de la displasia coxofemoral sería bastante elevada $(37 / 60=61.7 \%)$ considerando los datos registrados internacionalmente. Se demostró que no existe diferencia estadística significativa entre la frecuencia de presentación por sexos; que la enfermedad se presenta mayormente de modo bilateral, y dicho modo estaría estadísticamente asociado con la presencia de signos clínicos. Asimismo, se pudo observar que solo el $48.64 \pm 16.10 \%$ de los ejemplares afectados presentaron sintomatología clínica y que aparentemente no existe predisposición en el modo, grado y/o frecuencia de

\footnotetext{
'Práctica privada

${ }^{2}$ Clinica de Animales Menores - FMV - UNMSM

${ }^{3}$ Universidad Catolica de Chile
} 
afección de alguna articulación coxofemoral en particular (derecha ó izquierda). Se concluye que el estudio radiológico es indispensable y fundamental para establecer un diagnóstico detallado, preciso y definitivo de la displasia de cadera canina.

Palabras clave: Displasia de cadera, caninos, radiología, ángulo de Norberg, articulación coxofemoral.

\section{Trowhent}

La displasia de cadera es una anomalía en el desarrollo de la articulación coxofemoral que conduce a su inestabilidad, debido a una deficiente congruencia entre la cabeza femoral y el acetábulo, lo que se denomina "Laxitud articular" (Riser, 1996; Alexander, 1992). Este fenómeno conlleva a un movimiento anormal de la cabeza femoral, que genera diversos grados de subluxación e incluso luxación articular (Johnston, 1992).

Esta enfermedad es hereditaria y se presenta desde las primeras etapas de la vida hasta la adultez. La expresión clínica y el desarrollo de la misma son dependientes de factores ambientales, principalmente sobrecarga de la articulación en las primeras semanas de vida (Richardson, 1992).

La displasia de cadera es una enfermedad que se presenta con mucha frecuencia en la población canina (Málaga, 1966), afecta con mayor frecuencia a las razas grandes, bien alimentadas y de rápido crecimiento (Pedersen et al., 1992), y se ha diagnosticado en más de 60 razas (Corley, 1992).

Este estudio tiene el objetivo de establecer la frecuencia de presentación de la displacia de cadera en caninos de la raza Labrador Retriever, cuya crianza ha sido masificada intensamente en los últimos años.

\section{Materales re Mrtodos}

Se utilizaron 60 caninos ( 30 machos y 30 hembras) de la raza Labrador Retriever, de edades comprendidas entre 3 y 96 meses, los cuales acudieron a la Clínica de Animales Menores de la Facultad de Medicina
Veterinaria de la Universidad Nacional Mayor de San Marcos para diversos tipos de atención médico veterinaria. Previa anuencia de los dueños se obtuvieron las placas radiográficas de los caninos, todos ellos pertenecían al sector de Lima Metropolitana.

Se evaluaron radiológicamente según el método de Norberg las caderas de $60 \mathrm{ca}-$ ninos de la raza Labrador Retriever de ambos sexos (30 machos y 30 hembras), y se les agrupó en cinco categorías correspondientes a los diferentes grados establecidos por la norma internacional de la Federación Cinológica Internacional (FCI) para displasia de cadera.

Para facilitar la obtención de las radiografias y con ello obtener una buena calidad de imagen, los ejemplares fueron sedados con maleato de acepromacina en dosis de 0.5 $\mathrm{mg} / \mathrm{kg}$ por vía endovenosa.

Los animales fueron ubicados en posición decúbito dorsal con la región dorsotorácica situada en el posicionador para restringir el movimiento rotatorio de lateralidad. Los miembros torácicos se proyectaron en dirección craneal, ubicando la cabeza entre ellos. Los miembros pélvicos se traccionaron en dirección caudal, llevándolos a una posición en que el eje óseo femoral se dispuso paralelo al chasis radiográfico y a la vez se ubicaron paralelos entre sí. La pelvis se mantuvo en posición paralela con respecto al chasis radiográfico con el fin de obtener simetría entre los coxales (Shively, 1993; Carlson, 1977).

La técnica radiográfica empleó 5 miliAmperios-segundos ( $\mathrm{mA}-\mathrm{s})$ y el Kilovoltaje-potencia (Kv-P) se fijó de acuerdo al espesor de la región pélvica. Las 
películas radiográficas se revelaron manualmente en estanque, permaneciendo alrededor de 50 segundos en liquido revelador, igual tiempo en enjuague y 15 minutos a más en el líquido fijador. Luego se enjuagaron por última vez y se dejaron secar a temperatura ambiente.

La evaluación radiográfica se realizó a través de la determinación del ángulo de Norberg. Las radiografías se evaluaron sobre negatoscopio y luego fueron sometidas a la medición del ángulo de Norberg para cada articulación. Esta medición consistió en fijar los puntos centrales de cada cabeza femoral y unirlos con una línea recta; luego se marcaron los ángulos externos del borde acetabular craneal que sirvió como referencia para trazar una línea tangencial a los puntos anteriormente descritos. Se procedió a medir el ángulo que se orienta craneomedialmente. Los ejemplares con un ángulo inferior a 105 grados se consideraron positivos. La clasificación de la enfermedad se realizó, de acuerdo a la norma de la Federación Cinológica Internacional (F.C.I.), que considera los siguientes grados:

$\begin{array}{lll}\text { Grado } 0: & \begin{array}{l}\text { Normal }\left(105^{\circ} \text { a más) libre de }\right. \\ \text { displasia. }\end{array} \\ \text { Grado 0 a 1: } & \begin{array}{l}\left.\text { Transición (<a } 105^{\circ}\right) \text { sospe- } \\ \text { choso de displasia. }\end{array} \\ \text { Grado 1 : } & \text { Ligera }\left(100^{\circ} \text { a más) }\right. \\ \text { Grado 2 : } & \text { Media }\left(90^{\circ} \text { a más) }\right. \\ \text { Grado } 3 \text { y 4: } & \text { Grave }\left(90^{\circ} \text { a menos }\right)\end{array}$

Para efectos de la realización de los análisis estadísticos correspondientes, se crearon las cinco siguientes categorías, relacionadas con los diferentes grados de la enfermedad antes descritos:

$\begin{array}{ll}\text { Categoría A } & \begin{array}{l}\text { : Ejemplares con grado } 0 \\ \text { (normal) }\end{array} \\ \text { Categoría B }: \begin{array}{l}\text { Ejemplares con grado 0 a } 1 \\ \text { (transición o sospechoso) }\end{array} \\ \text { Categoría C : } \begin{array}{l}\text { Ejemplares con grado 1 (en- } \\ \text { fermedad ligera) }\end{array} \\ \text { Categoria D : } \begin{array}{l}\text { Ejemplares con grado 2 (en- } \\ \text { fermedad media) }\end{array} \\ \text { Categoría E } \quad \begin{array}{l}\text { Ejemplares con grado } 3 \text { y } 4 \\ \text { (enfermedad grave) }\end{array}\end{array}$

En el presente trabajo se consideraron como afectados a partir de la Categoría B, que pertenecen a la forma de transición (Grado 0 a 1) según la norma internacional de la F.C.I. (Cuadros 1, 2, 3 y 4).

A partir de los datos obtenidos se obtuvo la frecuencia de presentación de displasia de cadera en el total de las muestras y según el sexo; la asociación entre presencia de displasia de cadera y el sexo (chi cuadrado); y la distribución de los casos de displasia coxofemoral de acuerdo al modo de afección (unilateral para una sola cadera afectada y bilateral para ambas caderas afectadas) (Chi cuadrado); la asociación entre la presencia de signos clínicos y el modo de afección de la enfermedad (Chi cuadrado); la asociación entre la articulación coxofemoral afectada y los distintos grados de displasia de cadera (Chi cuadrado), adicionalmente, se determinó la correlación entre la edad de los animales y el grado de displasia coxofemoral (prueba no paramétrica de correlación de Spearman).

\section{mothong}

Se observó una frecuencia de presentación de displasia coxofemoral de $61.7 \pm$ $12.3 \%$ (Cuadro 1), que resulta elevada en relación con los registros internacionales de la Orthopedic Foundation for Animals, que durante 17 años y medio (1974-1991) registró 44009 caninos de la raza Labrador Retriever, obteniendo una frecuencia de presentación de la enfermedad de $14.6 \%$. (Corley, 1992).

Por otro lado, no se encontró asociación estadística entre la presentación de la enfermedad y el sexo. Además la evaluación de riesgo relativo fue de 1.15 (con un intervalo de confianza que va desde 0.41 hasta 3.26), lo cual confirmaría que el sexo no representa un factor de riesgo para la presentación de la enfermedad. 
Cuadro 1. Frecuencia de presentación de displasia de cadera en caninos de la raza Labrador Retriever

\begin{tabular}{lccl}
\hline Sexo & $\begin{array}{c}\text { Animales muestreados, } \\
\mathrm{n}\end{array}$ & $\begin{array}{c}\text { Animales afectados, } \\
\mathrm{n}\end{array}$ & Porcentaje \\
\hline Machos & 30 & 18 & $60.0^{*} \pm 17.53$ \\
Hembras & 30 & 19 & $63.3^{*} \pm 17.25$ \\
\hline Total & 60 & 37 & $61.7 \pm 12.30$ \\
\hline
\end{tabular}

${ }^{*}$ No existe diferencia estadística significativa

Tampoco se encontró asociación estadística entre la frecuencia de presentación y el modo de afección de la enfermedad según el número de articulaciones coxofemorales afectadas (uni o bilateral). En nuestros resultados se puede observar que la frecuencia de presentación de displasia coxofemoral de modo bilateral $59.5 \pm 20.5 \%$ es más común que el modo unilateral $40.5 \pm$ $24.8 \%$ coincidiendo con lo señalado por Riser (1996) y Fry y Clark (1992). (Cuadro 2).

Cuadro 2. Distribución de los casos de displasia según el modo de afección en caninos de la raza Labrador Retriever

Modo de afección Animales afectados, $\quad \underset{n}{\text { Animales muestreados, }} \quad$ Porcentaje

$\begin{array}{llll}\text { Unilateral } & 15 & 60 & 40.5 \pm 24.80 \\ \text { Bilateral } & 22 & 60 & 59.5 \pm 20.50\end{array}$

$\begin{array}{lrrr}\text { Total } & 37 & 60 & 100.0\end{array}$

Se observó una asociación estadística significativa entre el modo de presentación (displasia coxofemoral uni y bilateral) y la presencia de signos clínicos; lo que indicaría que el modo de presentación es un importante factor de riesgo para la presentación de signos clínicos (Cuadro 3).

De igual modo el estudio de riesgo relativo, que dio como resultado 8.57 (IC $=1.82$ 40.45 ), lo que significa que por cada animal con displasia coxofemoral de modo unilateral y con presencia de signos clínicos habría nueve animales afectados por el modo bilateral y con presencia de signos clínicos. Por lo tanto existe un mayor riesgo de observar signos clínicos por efecto del modo de afección (Cuadro 3).

En el presente experimento el $48.64 \pm$ $16.10 \%$ de los animales afectados uni o bilateralmente presentó sintomatología clínica.

Se puede apreciar que la enfermedad se puede presentar ya sea en la articulación coxofemoral derecha ó izquierda, con una frecuencia y grado similar. El mayor porcentaje tanto para el lado derecho $50 \pm 17.89$ como para el lado izquierdo $51.67 \pm 17.59 \mathrm{se}$ presentó en el "grado 0" (categoría A), que 
Cuadro 3. Frecuencia de presentación de signos clínicos en caninos radiológicamente positivos a displasia de cadera de la raza Labrador Retriever

\begin{tabular}{lccc} 
Modo de afección & $\begin{array}{c}\text { Animales afectados, } \\
\mathrm{n}\end{array}$ & $\begin{array}{c}\text { Presencia de signos } \\
\text { clínicos, } \mathrm{n}\end{array}$ & Porcentaje \\
\hline $\begin{array}{l}\text { Unilateral } \\
\text { Bilateral }\end{array}$ & 15 & 3 & $20.00 \pm 20.24$ \\
\hline Total & 22 & 15 & $68.18 \pm 09.90$ \\
\hline
\end{tabular}

corresponde a las articulaciones coxofemorales con $105^{\circ}$ o más según el método de Norberg y que son consideradas normales (Cuadro 4).

Dentro de los positivos, se puede observar grado 0 a 1 (categoría $B$ ), se presenta en menos porcentaje $8.33 \pm 32.69$ tanto para el lado derecho como para el lado izquierdo.

En los grados 1 y 2 (categorías $\mathrm{C}$ y D), que representan las formas de displasia de cadera Ligera y Media, respectivamente, obtuvieron los mismos porcentajes 13.33 \pm 23.55 para ambas articulaciones. Por último, en el grado 3 y 4 (categoría E) que representa la forma Grave de la enferme- dad, se observó $15 \pm 23.33 \%$ y $13.33 \pm$ $23.55 \%$ para el lado derecho e izquierdo respectivamente; no existiendo diferencia significativa entre la frecuencia de presentación entre ambas articulaciones. (Cuadro 4).

Aparentemente, no existiría correlación alguna entre el grado de afección de la enfermedad y la edad.

\section{Hewtin}

La frecuencia de la displasia coxofemoral fue aproximadamente cuatro veces mayor que la registrada internacionalmente por la Orthopedic Foundation for Animals

Cuadro 4. Clasificación de las articulaciones coxofemorales según los grados de afección de acuerdo a la norma internacional de la federación cinológica internacional $(\mathrm{FCl})$ en caninos de la raza Labrador Retriever

\begin{tabular}{lcccc}
\hline \multirow{2}{*}{$\begin{array}{c}\text { Clasificación en } \\
\text { grados según la norma de } \\
\text { la FCI* }\end{array}$} & \multicolumn{4}{c}{ Articulación coxofemoral } \\
\cline { 2 - 5 } \multicolumn{1}{c}{ Lado derecho } & \multicolumn{2}{c}{ Lado izquierdo } \\
\cline { 2 - 5 } & $\mathrm{n}$ & $\%$ & $\mathrm{n}$ & $\%$ \\
\hline Grado 0 & 30 & $50 \pm 17.89$ & 31 & $51.67 \pm 17.59$ \\
Grado 0 a 1 & 5 & $8.33 \pm 32.69$ & 5 & $8.33 \pm 32.69$ \\
Grado 1 & 8 & $13.33 \pm 23.55$ & 8 & $13.33 \pm 23.55$ \\
Grado 2 & 8 & $13.33 \pm 23.55$ & 8 & $13.33 \pm 23.55$ \\
Grado 3 y 4 & 9 & $15 \pm 23.33$ & 8 & $13.33 \pm 23.55$ \\
\hline Total & 60 & 100 & 60 & 100 \\
\hline
\end{tabular}

* Federación Cinológica Internacional 
(O.F.A.). La baja frecuencia de presentación publicada por la OFA. se debería en primer lugar a que los criadores y propietarios suelen enviar sólo las radiografías de los ejemplares con mayores probabilidades de certificarse como normales. Por otra parte, se estima que sólo la mitad de los propietarios que solicitan los estudios radiográficos a dicha entidad, envían las placas a la misma para que se realice la interpretación oficial. Es por ello que Smith y Mckelvie (1997) confirman que las cifras publicadas por la OFA serían dos a tres veces mayores.

Así mismo, en varios estados de los Estados Unidos (lugar del estudio de la OFA) muchos clubes exigen que los caninos estén certificados como normales para ser registrados y luego poder ser considerados aptos como reproductores, lo que resulta en un programa de selección para disminuir los porcentajes de la frecuencia de presentación de la displasia coxofemoral. (Corley, 1992).

El sexo no es un factor de riesgo para la presentación de la enfermedad; por lo tanto, se concluye que no existiría ninguna predisposición significativa por sexo, descartando así la teoría de Snavely (1978) que afirma que las hembras muestran mayor incidencia que los machos. Al respecto Smith y McKelvie, 1997; Riser 1996; Alexander 1992; Corley 1992; Pedersen, et al. 1992, sostienen que no existe una diferencia significativa entre sexos.

Fry y Clark (1992) mencionan que el $93 \%$ de los casos positivos son bilaterales, pero a veces solamente se presenta sintomatología clínica en la articulación coxofemoral más afectada. Esto se debería a que el dolor de la articulación menos afectada sería enmascarado por el de mayor dolor producido en la otra articulación, la cual estaría más deteriorada, provocando solamente la claudicación del miembro respectivo.

El porcentaje de animales afectados que presentó sintomatología clínica fue de 48.64, observándose que no todos los animales enfermos muestran síntomas clínicos. Esto podría deberse a que los animales utilizados en el presente experimento no fueron muestreados específicamente por presentar displasia coxofemoral. Además, según Fry y Clark (1992) el resto de los animales afectados podrían encontrarse en la etapa intermedia de la enfermedad, durante la cual no se observa sintomatología clínica. Dicha etapa puede durar meses o años y los caninos pueden caminar o correr sin dolor.

Aunque se obtuvo el mayor porcentaje en las articulaciones coxofemorales "normales" (buena coaptación articular, ángulo de Norberg igual a 105 grados o más, congruencia entre las superficies articulares) ya sea derecha o izquierda (Cuadro 4), no significa que la mayoría de caninos estuvieran sanos, ya que a pesar de presentar una articulación coxofemoral normal, la enfermedad podía estar presente en la otra articulación, condición suficiente para que un animal sea considerado displásico.

No se observó que correlación entre el grado de afección de la enfermedad y la edad, lo que corroboraría la información que presentan Riser (1996), Alexander (1992), Fry y Clark, (1992), Morgan (1992), otros; que señalan que la enfermedad se puede presentar en animales jóvenes como en viejos, pero lo que difiere es el tipo de manifestación de los signos clínicos, es decir que mientras un animal joven cojea después del ejercicio, el animal viejo muestra renuencia al ejercicio, lo cual sería resultado de la degeneración articular.

\section{ermatoris:}

A partir del presente estudio radiológico se determinó que la frecuencia de presentación de displasia coxofemoral es de $61.7 \%$, la que es muy elevada considerando los datos registrados internacionalmente y que la presentación de la enfermedad no está asociada al sexo. 
En este trabajo, la enfermedad se presentó mayormente de modo bilateral $(59.5 \%)$, y acompañado de sintomatología clínica $(68.18 \%)$ y sólo el $48.64 \%$, de los ejemplares afectados presentaron sintomatología clínica y aparentemente, no existe predisposición en el modo, grado y/o frecuencia de afección por alguna articulación coxofemoral en particular (ya sea en el lado derecho ó izquierdo).

No se obtuvo correlación entre la edad de los ejemplares y el grado de afección de la enfermedad.

\section{Theraluma Clada}

1. Alexander, J. 1992. The pathogenesis of canine hip dysplasia. Veterinary Clinics of North America: Small Animal Practice. EEUU.22 (3):503-511

2. Carlson, W. D. 1977. Carlson veterinary radiology. $3^{\mathrm{a}}$ edition., p. 130-338. Lea \& Febiger. Philadelphia-USA.

3. Corley, E. 1992. Role of the Orthopedic Foundation for Animals in the control of canine hip dysplasia. Veterinary Clinics of North America: Small Animal Practice. EEUU. 22(3):579-593.

4. Fry, T. y D. Clark. 1992. Canine hip dysplasia: clinical signs and physical diagnosis. Veterinary Clinics of North America: Small Animal Practice. EEUU. 22(3):551-557.

5. Johnston, S. 1992. Conservative and medical management of hip dysplasia. Veterinary Clinics of North America: Small Animal Practice. EEUU. 22(3):595-605.
6. Málaga, H. 1966. Displasia coxofemoral en caninos de la policía militar. Tesis Bachillerato. Fac. Med. Vet. Univ. Nac. Mayor San Marcos, Lima. 44p.

7. Morgan, S. 1992. The pathology of canine hip dysplasia. Veterinary Clinics of North America: Small Animal Practice. EEUU. 22(3):541-550.

8. Pedersen, N. C.; A. Wind; J. P. Morgan y R.R. Pool. 1992. Enfermedades articulares del perro y el gato. En: Tratado de medicina interna veterinaria. Enfermedades del perro y del gato. Tomo III, Cap. 120, Ed. S. Ettinger. $3^{\mathrm{a}}$ edición. p. 2449-2498. Editorial Intermédica, Buenos Aires-Argentina.

9. Richardson, D. 1992. The role of nutrition in canine hip dysplasia. Veterinary Clinics of North America: Small Animal Practice. EEUU. 22(3):529-539.

10. Riser, W. H. 1996. Canine hip dysplasia. En: Current techniques in small animal surgery. Cap.112, Ed. J.Bojrab. $3^{\text {a }}$.Ed. p. 821828. Lea \& Febiger. Philadelphia-USA.

11. Shively, M. J. 1993. Anatomía veterinaria básica comparativa y clínica. p. 121-122. Editorial El Manual Moderno. México D.F.

12. Smith, C. K. y P.J. McKelvie. 1997. Conceptos actuales en el diagnóstico de displasia de cadera en perros. En: Terapeútica veterinaria de pequeños animales. $12^{\mathrm{a}}$ Ed., p. 1272-1281. Ed. R. Kirk y J. Bonagura. Editorial McGrawHill. México.

13. Snavely. 1978. Displasia de las ancas (articulación coxofemoral). En: Traumatología y Ortopedia Canina. Ed. W. Whittick. p.249-279. Editorial Aedos. Barcelona-España. 